\title{
VELOCIMETRIA DOPPLER NO PERÍODO NEONATAL EM RECÉM-NASCIDOS A TERMO PEQUENOS PARA IDADE GESTACIONAL
}

\author{
Iracema Augusta Carvalho Cortez Muniz', Abimael Aranha Netto², \\ Vanda Maria Gimenes Gonçalves ${ }^{3}$
}

\begin{abstract}
RESUMO - Este estudo teve como objetivo avaliar o fluxo sanguíneo cerebral em recém-nascidos a termo pequenos para idade gestacional, utilizando ultra-sonografia Doppler craniana ao nascimento. 0 estudo foi desenvolvido no CAISM/UNICAMP (Centro de Saúde Terciário para Mulheres). Foram selecionados sessenta recém-nascidos e divididos em 2 grupos: 36 adequados para idade gestacional (AIG) e 24 pequenos para idade gestacional (PIG). Foi realizada a avaliação de ultra-sonografia Doppler craniana nos grupos, entre 24-48 horas após o nascimento. A velocidade de fluxo sanguíneo cerebral foi menor no grupo PIG na artéria cerebral anterior (ACA). As medidas Doppler foram estatisticamente diferentes entre os grupos apenas para valores relacionados à velocidade de fluxo de pico sistólico (VFPS) e velocidade de fluxo médio (VFM) na ACA. Não houve diferenças significantes para nenhum dos parâmetros avaliados de velocidade de fluxo na artéria cerebral média (ACM). Concluiu-se que recém-nascidos PIG apresentaram VFPS e VFM significativamente menores apenas na ACA. A adequação peso/ idade gestacional, presença de policitemia neonatal e valores de pressão arterial média estiveram estatisticamente associados a VFM na ACA. A presença de sofrimento fetal, valores de pressão arterial média e hábito de fumar durante a gestação estiveram estatisticamente associados a VFM na ACM.
\end{abstract}

PALAVRAS-CHAVE: Doppler-ultra-sonografia craniana, desnutrição intra-uterina, recém-nascido.

\section{Neonatal Doppler velocimetry in full term small-for-gestational age newborns}

\begin{abstract}
This study aimed to evaluate the cerebral blood flow of full term small-for-gestational age newborns, using cranial ultrasound Doppler at birth. This study was performed at CAISM/UNICAMP (Tertiary Health Center for Women). Sixty term newborns were selected and divided in two groups: appropriate-for-gestational age (AGA) (36 neonates) and small-for-gestational age (SGA) (24 neonates). Cranial ultrasound Doppler evaluation was performed on both groups, between 24 and 48 hours after birth. Cerebral blood flow velocity (CBFV) was lower in the small-for-gestational age group, in the anterior cerebral artery (ACA). Doppler measurements were different statistically between the groups only for values related to peak systolic flow velocity (PSFV) and mean flow velocity (MFV) in the ACA. There was no significant difference for any evaluated parameters of flow velocity in the middle cerebral artery (MCA). It was concluded that SGA newborns showed PSFV and MFV significantly reduced only in the ACA. Weight/gestational age, neonatal polycythemia and mean arterial blood pressure values were statistically related to MFV in the ACA. In presence of fetal suffering, mean arterial blood pressure values and smoking in the pregnancy were statistically related to MFV in the MCA.
\end{abstract}

KEY WORDS: cranial ultrasound Doppler, intrauterine malnutrition, newborn.

A desnutrição intra-uterina representa grande problema de saúde pública para países emergentes como o Brasil, sendo responsável direta por parcela significativa da morbi-mortalidade no período neonatal ${ }^{1,2}$. A fisiopatogenia dessas manifestações mórbidas continua pouco esclarecida, podendo depen- der, entre outras, de etiologia específica ${ }^{3}$, do grau ou do tempo de duração da desnutrição durante a gestação $0^{2,4}$. Embora haja controvérsia sobre o tipo e a magnitude do dano causado pela desnutrição ao sistema nervoso central do recém-nascido (RN), parece haver pouca dúvida quanto ao impacto sobre o

Faculdade de Ciências Médicas da Universidade Estadual de Campinas/São Paulo, Campinas SP, Brasil (FCM-UNICAMP): ${ }^{1}$ Mestre em Pediatria; ${ }^{2}$ Professor Assistente Doutor do Departamento de Pediatria; ${ }^{3}$ Professor Assistente Doutor do Departamento de Neurologia. Pesquisa financiada pela Fundação de Amparo à Pesquisa do Estado de São Paulo (FAPESP). Processo 00/02884-3

Recebido 27 Novembro 2002, recebido na forma final 28 Abril 2003. Aceito 14 Maio 2003.

Dra. Vanda Maria Gimenes Gonçalves - Departamento de Neurologia, FCM-UNICAMP - Caixa Postal 6111 - 13081-970 Campinas SP Brasil.E-mail: vandagg@uol.com.br 
desenvolvimento neuropsicomotor em crianças com retardo de crescimento intra-uterino $(\mathrm{RCIU})^{5,6}$, sendo inegável a existência de índices mais baixos de desempenho motor e de percepção em recém-nascidos pequenos para idade gestacional (RN PIG) ${ }^{6}$.

A fisiopatogenia dessas diferenças não está esclarecida, podendo estar vinculada, entre alternativas, a fenômenos circulatórios, visto haver alguns estudos que estabeleceram correlação entre desenvolvimento neurológico alterado na infância e alterações hemodinâmicas cerebrais no período neonatal, particularmente em relação à isquemia cerebral em RN prematuros com desnutrição intra-uterina, sugerindo, portanto, a existência de um fenômeno de má adaptação circulatória ? .

Baseado em tais evidências, o presente estudo foi concebido com o objetivo de avaliar a velocidade de fluxo sanguíneo cerebral (FSC) através do módulo Doppler realizado entre 24-48 horas de vida e a possível associação com algumas variáveis maternas e perinatais em RN a termo PIG, comparando os resultados com aqueles obtidos em RN adequados para idade gestacional (RN AIG), com a finalidade de utilizá-lo futuramente como instrumento de predição do risco no período neonatal para alterações no território vascular cerebral correspondente e o prognóstico neurológico em médio e longo prazo.

\section{MÉTODO}

Após assinatura do Termo de Consentimento Livre e Esclarecido, foram analisados prospectivamente $60 \mathrm{RN}$ de termo (RNT), nascidos vivos no Serviço de Neonatologia do Centro de Atenção Integral à Saúde da Mulher (CAISM) da Universidade Estadual de Campinas (UNICAMP), no período de março de 2000 a outubro de 2001 . O grupo 1 foi constituído de 36 RNT AIG e o grupo 2 por 24 RNT PIG de acordo com as curvas de peso ao nascer de Lubchenco et al. ${ }^{8}$. Utilizaram-se como critérios de inclusão: RNT, nascidos vivos e mantidos em alojamento conjunto, sendo alimentados preferencialmente por leite materno exclusivo. Foram excluídos os RN com malformações congênitas diagnosticadas no período neonatal e aqueles com necessidade de internação em unidade neonatal no período de avaliação.

Utilizou-se a adequação peso/idade gestacional como variável independente, sendo classificado como AIG o RN com peso de nascimento entre o percentil 25 e 90 do valor de referência e como PIG aquele com peso de nascimento abaixo do percentil 10. O peso foi obtido em gramas, logo após o nascimento, mensurado em balança eletrônica com precisão de 10 gramas aferida regularmente.

As variáveis dependentes foram representadas pelas medidas de velocidade de FSC na artéria cerebral anterior ( $A C A)$ e artéria cerebral média ( $A C M$ ) medidas por ecografia Doppler entre 24 - 48 horas de vida. Mensurouse a velocidade de fluxo no pico sistólico (VFPS); velocida- de de fluxo no final da diástole (VFFD); velocidade média de fluxo (VMF); índice de resistência de Pourcelot (IR), definido como VFPS-VFFD/VFPS e índice de pulsatilidade (IP), definido como VFPS-VFFD/VMF. Essas medidas foram realizadas durante a internação do RN no Serviço de Neonatologia, por investigador previamente treinado. Utilizou-se equipamento de ultra-som Aloka, dotado de Doppler pulsado, transdutor de $5 \mathrm{Mhz}$ e filtro de $200 \mathrm{~Hz}$. As ACA e ACM foram localizadas anatomicamente pelo módulo bidimensional do ultra-som e detectadas as ondas de fluxo sanguíneo através do módulo de Doppler pulsado ${ }^{9}$. A ACA foi visualizada através da janela transfontanelar anterior, na região pericalosa e a ACM foi aferida na região temporal, ao nível do polígono de Willis.

As variáveis de controle incluíram fatores maternos: idade, grau de paridade, patologias crônicas e/ou gestacionais e tabagismo durante a gestação, e fatores perinatais: sofrimento fetal, características do líquido amniótico, tipo de alteração de avaliação Doppler intra-útero, tipo de parto (normal e cesariana), Índice de APGAR ao $1^{\circ}$ e $5^{\circ}$ minutos, idade gestacional em semanas completas de gestação, avaliada conforme método proposto por Capurro et al. ${ }^{10}$. Tolerou-se diferença de mais ou menos uma semana, com o dado obtido através de amenorréia materna e/ou ultrasonografia fetal, realizada até 24 semanas de gestação; proporcionalidade corporal: definida pela relação entre o peso e o comprimento ao nascer, calculado segundo a fórmula de Rohrer ${ }^{11}=$ g. 100/cm ${ }^{3}$ (IPR), caracterizados em assimétricos (crianças com IPR < P10 para a IG) ou simétricos (crianças com IPR $\geq$ P10 para a IG) e pressão arterial média (PAM) por método de oscilometria não invasiva.

Os fatores maternos foram obtidos a partir de entrevistas e de informações constantes no prontuário médico. Todos os dados foram coletados utilizando-se uma planilha pré-codificada, posteriormente armazenados em meio eletrônico através do programa Epi-Info 6. A análise estatística foi realizada através do programa SAS versão 8.1.

As variáveis foram analisadas por frequência, mediana e desvio-padrão. Os valores de FSC entre RN PIG e AIG foram analisados pelo teste $t$ de Student ou Mann-Whitney, para amostras independentes. Para avaliar a influência das variáveis independentes (AIG/PIG, sexo, peso ao nascer, presença/ausência de policitemia neonatal, IPR, IG, presença/ausência de sofrimento fetal, PAM e tipo de parto) nos valores de velocidade de FSC, utilizou-se como variável dependente apenas a VMF nas ACA e ACM por representar a integração da curva da área abaixo de cada ciclo cardíaco e estar melhor associado ao fluxo sanguíneo real. Para cada variável resposta de interesse, fez-se a análise de regressão linear univariada e depois foram analisadas conjuntamente através de análise múltipla, sendo descritas apenas aquelas com significação estatística. Considerou-se em $5 \%(p<0,05)$ o limite de significação estatística.

Foram cumpridos rigorosamente as disposições e os princípios da Declaração de Helsinque, emendada na África do Sul (1996) e obedecidos os princípios da Resolução 196 do Conselho Nacional de Saúde (Inf. Epidem. do SUS, 
Brasil, Ano V, № 2, 1996). 0 estudo obteve a aprovação do Comitê de Ética e Pesquisa da Instituição e da Fundação de Amparo à Pesquisa do Estado de São Paulo (FAPESP).

\section{RESULTADOS}

A amostra analisada constituiu-se de 60 neonatos de termo, sendo classificados como AIG, 36 e como PIG, 24. As características clínicas gerais da população estudada foram semelhantes, exceto o peso de nascimento que foi significativamente menor no grupo 2: a mediana do peso dos RN classificados como AIG foi de $3227 \mathrm{~g}$ e a mediana dos PIG foi de $2375 \mathrm{~g}(\mathrm{p}<0,01)$. O peso mínimo observado foi $2125 \mathrm{~g}$ e o máximo $3710 \mathrm{~g}$. (Tabela 1 ).
A análise da proporção corporal pelo método de Rohrer mostrou que $75 \%$ dos PIG eram simétricos, ou seja, apresentou padrão proporcionado de crescimento intra-útero $(p<0,01)$. A policitemia (hematócrito periférico $\geq 65 \%$ ) esteve presente em $17 \%$ dos PIG $(p=0,02)$.

As variáveis maternas estudadas estão dispostas na Tabela 2. A maioria dos fatores maternos não exibiu diferença estatisticamente significativa, com exceção da idade materna e tabagismo durante a gestação. Observou-se que eram filhos de mães com idade entre 18 e 25 anos, $85 \%$ dos neonatos; entretanto, entre os PIG eram filhos de mães adolescentes, $21 \%$ e de mães com idade superior a 35 anos,

Tabela 1. Perfil da população estudada.

\begin{tabular}{|c|c|c|c|c|c|c|}
\hline \multirow[b]{3}{*}{ Dados do RN } & \multirow[b]{3}{*}{$n(\%)$} & \multicolumn{4}{|c|}{ Estadonutricional } & \multirow[b]{3}{*}{$p$ valor } \\
\hline & & \multicolumn{2}{|c|}{$A \mid G$} & \multicolumn{2}{|c|}{$P \mid G$} & \\
\hline & & $n$ & (\%) & $n$ & $(\%)$ & \\
\hline Sexo & & & & & & 0,42 \\
\hline Masculino & $22(37)$ & 15 & $(42)$ & 7 & (29) & \\
\hline Feminino & $38(63)$ & 21 & $(58)$ & 17 & (71) & \\
\hline Cor & & & & & & 0,59 \\
\hline Branca & $24(40)$ & 13 & (36) & 11 & $(46)$ & \\
\hline Não branca & $36(60)$ & 23 & $(64)$ & 13 & (54) & \\
\hline Peso (gramas) & & & & & & $<0,01$ \\
\hline Mínimo & 2125 & 2765 & & 2125 & & \\
\hline Mediana & 3042 & 3227 & & 2375 & & \\
\hline Máximo & 3710 & 3710 & & 2655 & & \\
\hline APGAR $1^{\circ}$ minuto & & & & & & 1,00 \\
\hline$<7$ & $3(5)$ & 2 & (6) & 1 & $(4)$ & \\
\hline$\geq 7$ & $57(95)$ & 34 & (94) & 23 & (96) & \\
\hline APGAR $5^{\circ}$ minuto & & & & & & 1,00 \\
\hline$<7$ & $0 \quad(0)$ & 0 & $(0)$ & 0 & $(0)$ & \\
\hline$\geq 7$ & $60(100)$ & 36 & $(100)$ & 24 & $(100)$ & \\
\hline IG (semanas) & & & & & & 0,67 \\
\hline 37 & 3 (5) & 1 & (3) & 2 & (8) & \\
\hline 38 & $11(18)$ & 8 & $(22)$ & 3 & (13) & \\
\hline 39 & $19(32)$ & 12 & (33) & 7 & (29) & \\
\hline 40 & $22(37)$ & 13 & (36) & 9 & (37) & \\
\hline 41 & $5 \quad(8)$ & 2 & (6) & 3 & (13) & \\
\hline ÍndicePonderal & & & & & & $<0,01$ \\
\hline Simétrico & $54(90)$ & 36 & $(100)$ & 18 & (75) & \\
\hline Assimétrico & $6(10)$ & 0 & $(0)$ & 6 & (25) & \\
\hline Policitemia & & & & & & 0,02 \\
\hline $\mathrm{Sim}$ & $4 \quad(7)$ & 0 & $(0)$ & 4 & (17) & \\
\hline Não & $55(93)$ & 35 & $(100)$ & 20 & (83) & \\
\hline
\end{tabular}

(a) Teste exato de Fisher; $n$, número de sujeitos; AIG, adequado para a idade gestacional; PIG, pequeno para a idade gestacional. 
$12 \%(p<0,01)$. Quanto ao grau de paridade, eram filhos de multíparas $50 \%$ dos PIG. Fumaram durante a gestação $25 \%$ das gestantes e eram filhos de mães fumantes $42 \%$ dos PIG $(p=0,03)$.

Sofrimento fetal esteve presente em $13 \%$ dos RN, com ocorrência semelhante nos dois grupos. Em relação às características do líquido amniótico, apresentaram-no claro, $75 \%$ dos RN, meconial fluido em $20 \%$ e meconial espesso em $5 \%$. O exame de Doppler intra-uterino foi realizado em $83 \%$ das gestantes e foi alterado em $16 \%$ dos casos (DIP II em 7 e bradicardia fetal em 1). O parto normal predominou na amostra estudada (68\%) e no grupo 2 (75\%).

A análise dos valores de velocidade de FSC em relação ao estado nutricional revelou que os valores medianos (VFPS, VFFD e VMF), mensurados na ACA foram sempre menores nos RN PIG, com significância estatística em relação às velocidades sistólica $(p=0,02)$ e média $(p=0.05)$ (Tabela 3$)$.

Resultados semelhantes foram observados nos valores médios de velocidade de FSC na ACM, com valores menores no grupo de RN PIG. Entretanto, as diferenças não foram significativas do ponto de vista estatístico. Da mesma forma, não foram observa-

Tabela 2. Perfil das gestantes.

\begin{tabular}{|c|c|c|c|c|c|c|}
\hline \multirow[b]{3}{*}{ Dados maternos } & \multirow[b]{3}{*}{$n(\%)$} & \multicolumn{4}{|c|}{ Estadonutricional } & \multirow[b]{3}{*}{$p$ valor ${ }^{(a)}$} \\
\hline & & \multicolumn{2}{|c|}{$A \mid G$} & \multicolumn{2}{|c|}{$P \mid G$} & \\
\hline & & $n$ & $(\%)$ & $n$ & $(\%)$ & \\
\hline \multicolumn{6}{|l|}{ Idade(anos) } & $<0,01$ \\
\hline$<18$ & $6(10)$ & 1 & (3) & 5 & $(21)$ & \\
\hline $18-35$ & $51(85)$ & 35 & (97) & 16 & (67) & \\
\hline$>35$ & $3(5)$ & 0 & $(0)$ & 3 & $(12)$ & \\
\hline \multicolumn{6}{|l|}{ Paridade } & 0,65 \\
\hline Nulípara & $4(7)$ & 2 & (6) & 2 & (8) & \\
\hline 1 & $20(33)$ & 13 & (36) & 7 & (29) & \\
\hline 2 & $11(18)$ & 8 & $(22)$ & 3 & (13) & \\
\hline 3 ou mais & $25(42)$ & 13 & (36) & 12 & $(50)$ & \\
\hline \multicolumn{6}{|l|}{ Patologias crônicas } & 0,68 \\
\hline Ausentes & $54(90)$ & 33 & $(92)$ & 21 & (88) & \\
\hline Presentes & $6(10)$ & 3 & (8) & 3 & $(12)$ & \\
\hline \multicolumn{6}{|c|}{ Patologias gestacionais } & 0,60 \\
\hline Ausentes & $31(52)$ & 20 & (56) & 11 & $(46)$ & \\
\hline Presentes & $29(48)$ & 16 & $(44)$ & 13 & $(54)$ & \\
\hline \multicolumn{6}{|l|}{ Tabagismo } & 0,03 \\
\hline Não & $45(75)$ & 31 & (86) & 14 & (58) & \\
\hline Sim & $15(25)$ & 5 & $(14)$ & 10 & $(42)$ & \\
\hline \multicolumn{6}{|l|}{ Sofrimento fetal } & 1,00 \\
\hline Ausente & $52(87)$ & 31 & (86) & 21 & (88) & \\
\hline Presente & $8(13)$ & 5 & (14) & 3 & $(12)$ & \\
\hline \multicolumn{6}{|l|}{ Líquido amniótico } & 1,00 \\
\hline Claro & $45(75)$ & 27 & (75) & 18 & $(75)$ & \\
\hline Mecônio espesso & $3(5)$ & 2 & $(6)$ & 1 & (4) & \\
\hline Mecônio fluido & $12(20)$ & 7 & (19) & 5 & $(21)$ & \\
\hline \multicolumn{6}{|c|}{ Doppler intra-uterino(b) } & 0,46 \\
\hline Normal & $42(84)$ & 27 & (87) & 15 & (79) & \\
\hline Alterado & $8(16)$ & 4 & (13) & 4 & $(21)$ & \\
\hline \multicolumn{6}{|l|}{ Tipo de parto } & 0,41 \\
\hline Normal & $41(68)$ & 23 & (64) & 18 & (75) & \\
\hline Cesariana & $19(32)$ & 13 & (36) & 6 & (25) & \\
\hline
\end{tabular}

(a) Teste exato de Fisher; $n$, número de sujeitos; AIG, adequado para a idade gestacional; PIG, pequeno para a idade gestacional; (b) Doppler intra-uterino não realizado em 10 casos, sendo 5 PIG e 5 AIG. 
das diferenças nos valores de IR e IP entre os grupos, seja na ACA ou ACM.

$\mathrm{Na}$ análise de regressão univariada, apenas a adequação peso/idade gestacional e a presença de policitemia neonatal estiveram relacionadas aos valores de velocidade média de fluxo na ACA e ACM. Entretanto, quando realizada análise de regressão múltipla, considerando-se como variável dependente a velocidade média de fluxo na artéria cerebral anterior observou-se que a adequação peso/idade gestacional $(p=0,04)$, presença de policitemia neonatal $(p=0,03)$ e valores de pressão arterial média $(p=0,01)$ foram estatisticamente associados aos seus valores de fluxo $\left(R^{2}=0,24\right)$ (Tabela 4).

Por outro lado, quando a variável dependente estudada foi a velocidade média de fluxo na artéria cerebral média observou-se fatores diferentes de associação $\left(R^{2}=0,16\right)$, como a presença de sofrimento fetal $(p=0,04)$, valores de pressão arterial média $(p=0,02)$ e o hábito de fumar durante a gestação $(p=0,04)$ (Tabela 5).

\section{DISCUSSÃO}

A análise dos valores do FSC em RN de termo demonstrou que os RN PIG da amostra apresentaram valores de FSC sistematicamente diminuídos tanto nos territórios da ACA como da ACM, seja pelas avaliações de velocidade sistólica, média ou diastólica; encontrou-se, entretanto, diferenças estatisticamente significativas apenas em relação às velocidades sistólica e média na ACA.

Alguns autores mostraram nas primeiras horas de vida, aumento do FSC entre PIG e diminuição nos valores dos IR, mimetizando a continuidade do fenômeno de 'preservação cerebral' encontrado no período fetal 12,13; outros não encontraram diferenças significativas ${ }^{14} \mathrm{ou}$, contrariamente, valores mais baixos de fluxo e elevação de resistência vascular em concordância com o presente estudo ${ }^{15}$.

Resultados tão opostos demonstram que provavelmente as populações estudadas sejam diversificadas, dificultando que se estabeleça explicação uni-

Tabela 3. Valores de fluxo sangüíneo cerebral, segundo o estado nutricional do $R N$.

\begin{tabular}{|c|c|c|c|c|c|c|}
\hline Variáveis & $\begin{array}{c}\text { Estado } \\
\text { nutricional }\end{array}$ & $n$ & mediana & (mínimo & máximo) & $P$ valor ${ }^{(a)}$ \\
\hline \multirow[t]{2}{*}{ VFPS ACA } & AIG & 36 & 31,73 & $(23,58$ & $46,61)$ & 0,02 \\
\hline & PIG & 24 & 27,52 & $(17,97$ & $48,85)$ & \\
\hline \multirow[t]{2}{*}{$V F F D A C A$} & AIG & 36 & 10,11 & $(4,49$ & $14,04)$ & 0,16 \\
\hline & PIG & 24 & 9,55 & $(5,62$ & $15,16)$ & \\
\hline \multirow[t]{2}{*}{ VMF ACA } & AIG & 36 & 19,43 & $(14,17$ & $27,88)$ & 0,05 \\
\hline & PIG & 24 & 17,05 & $(11,87$ & $30,39)$ & \\
\hline \multirow[t]{2}{*}{ IR ACA } & AIG & 36 & 0,663 & $(0,533$ & $0,759)$ & 0,47 \\
\hline & PIG & 24 & 0,644 & $(0,545$ & $0,750)$ & \\
\hline \multirow[t]{2}{*}{ IP $A C A$} & AIG & 36 & 1,096 & $(0,880$ & $1,481)$ & 0,62 \\
\hline & PIG & 24 & 1,094 & $(0,850$ & $1,357)$ & \\
\hline \multirow[t]{2}{*}{ VFPS ACM } & AIG & 36 & 49,98 & $(35,38$ & $99,95)$ & 0,39 \\
\hline & PIG & 24 & 49,13 & $(23,02$ & $86,47)$ & \\
\hline \multirow[t]{2}{*}{ VFFD ACM } & AIG & 36 & 13,48 & $(8,42$ & $47,17)$ & 0,58 \\
\hline & PIG & 24 & 12,64 & $(6,18$ & $23,58)$ & \\
\hline \multirow[t]{2}{*}{ VMF ACM } & AIG & 36 & 29,59 & $(20,08$ & $71,78)$ & 0,28 \\
\hline & PIG & 24 & 25,93 & $(12,29$ & $50,92)$ & \\
\hline \multirow[t]{2}{*}{ IR ACM } & AIG & 36 & 0,705 & $(0,523$ & $0,825)$ & 0,61 \\
\hline & PIG & 24 & 0,705 & $(0,592$ & $0,798)$ & \\
\hline \multirow[t]{2}{*}{ IP ACM } & AIG & 36 & 1,295 & $(0,735$ & $1,734)$ & 0,85 \\
\hline & PIG & 24 & 1,235 & $(0,963$ & $1,774)$ & \\
\hline
\end{tabular}

(a)Teste não-paramétrico de Mann-Whitney; ACA, artéria cerebral anterior; ACM, artéria cerebral média; VFPS, velocidade de fluxo no pico sistólico; VFFD, velocidade de fluxo no final da diástole; VMF, velocidade média de fluxo; IP, índice de pulsatilidade; IR, índice de resistência de Pourcelot. $n$, número de sujeitos; AIG, adequado para a idade gestacional; PIG, pequeno para a idade gestacional. 
versal para a relação fluxo-adequação peso/idade gestacional; além do que parte da literatura descrita anteriormente trate exclusivamente de recém-nascidos prematuros. Outro ponto a ser considerado é que o modelo de controle do FSC no período neonatal ainda é pouco definido e estudos experimentais demonstram que o FSC modifica-se continuamente e as mudanças podem ser decorrentes de uma série de fatores, entre eles, o amadurecimento cronológico e hemodinâmico, que geram variabilidade nos valores normais nas velocidades de fluxo. Assim, tem sido descrito que as velocidades podem variar com a idade gestacional, a idade pós-natal, o peso ao nascimento, o estado clínico, o uso de drogas, o hematócrito, a $\mathrm{pCO}_{2}$, a função cardiopulmonar, a pressão arterial sistêmica e a presença de doenças intracranianas, dentre outros. Além dessas variáveis, ainda podem existir diferenças em relação à forma de distribuição do fluxo arterial, como por exemplo, um predomínio hemisférico ${ }^{16}$.

Essa profusão de variáveis aumenta a dificuldade da comparação entre os estudos, pois dificilmente se podem confrontar as populações utilizadas, notadamente quando se trata de recém-nascidos de muito baixo peso, onde incide um grande número de patologias. Por outro lado, tem-se que perfusão cerebral no feto e o ritmo metabólico cerebral estão intimamente acoplados ${ }^{17}$. O transporte deste conceito para o período pós-natal imediato poderia subsi- diar uma hipótese para explicar a diferença de fluxo entre os grupos de RN desta pesquisa. Sob este ponto de vista, os requerimentos metabólicos dos grupos poderiam ser diferentes, caracterizando um fenômeno adaptativo de acoplamento requerimento metabólico-fluxo pela menor massa encefálica no grupo de menor peso e não um estado de diminuição anormal do FSC. No entanto, a falta de diferença de valores de fluxo na ACM nos RN analisados dificulta essa explanação; além disso, não se encontrou associação entre o padrão de crescimento intra-uterino principalmente em relação à simetria/assimetria, como se poderia esperar, embora os números avaliados no trabalho sejam pequenos para uma análise mais profunda a esse respeito.

É importante realçar que grande parte das crianças avaliadas nesta pesquisa apresentava padrão proporcionado de crescimento intra-útero, típico de países em desenvolvimento, podendo, portanto, diferenciar-se no que diz respeito ao fluxo cerebral de populações estudadas em outros países, onde predominam padrões assimétricos de crescimento, com forte componente de alteração placento-fetal, presença de sofrimento fetal e preservação do sistema nervoso, fenômenos pouco observados neste estudo. No sentido oposto, alguns estudos que utilizaram medidas seriadas de velocidade de fluxo tanto na ACA como na ACM, mostraram respostas diferentes em relação à desnutrição intra-uterina. Em

Tabela 4. Análise das variáveis perinatais em relação à velocidade média de fluxo na artéria cerebral anterior.

\begin{tabular}{lccccc}
\hline $\begin{array}{l}\text { Variável } \\
\text { independente }\end{array}$ & Classes & $\begin{array}{c}\text { Parâmetro } \\
\text { Estimado }\end{array}$ & Erro padrão & $t$ valor & $p$ valor \\
\hline Intercepto & - & 2,56671 & 0,12428 & 20,65 & $<0,01$ \\
Estado nutricional & PIG/AIG & 0,11438 & 0,05479 & 2,09 & 0,04 \\
Policitemia & sim/não & $-0,23566$ & 0,10412 & $-2,26$ & 0,04 \\
Pressão arterial média & Contínua & 0,00555 & 0,00211 & 2,62 & 0,01 \\
\hline
\end{tabular}

AIG, adequado para a idade gestacional; PIG, pequeno para a idade gestacional.

Tabela 5. Análise das variáveis perinatais em relação à velocidade média de fluxo na artéria cerebral média.

\begin{tabular}{lccccc}
\hline $\begin{array}{l}\text { Variável } \\
\text { independente }\end{array}$ & Classes & $\begin{array}{c}\text { Parâmetro } \\
\text { Estimado }\end{array}$ & Erro padrão & $t$ valor & $p$ valor \\
\hline Intercepto & - & 3,01966 & 0,14315 & 21,09 & $<0,01$ \\
Sofrimento fetal & sim/não & 0,19370 & 0,09662 & 2,00 & 0,04 \\
Pressão arterial média & contínua & 0,00637 & 0,00278 & 2,29 & 0,02 \\
Tabagismo & sim/não & $-0,15855$ & 0,07751 & $-2,05$ & 0,04 \\
\hline
\end{tabular}


RN PIG evidenciaram vasodilatação e aumento do fluxo na ACM e incapacidade de vasodilatar da ACA, em uma espécie de fenômeno de regionalização da perfusão que, em tese, também poderia justificar a predileção para fenômenos isquêmicos na região frontal nos recém nascidos com retardo de crescimento intrauterino ${ }^{18}$. No estudo atual não se conseguiu demonstrar tal efeito, embora tivesse havido nítida diferenciação entre o comportamento dos dois vasos.

Apesar de encontrar-se resultados de diminuição de fluxo entre crianças PIG em alguns estudos ${ }^{19,20}$, é senso comum, entre diversos autores, configurar para o PIG, mesmo após o nascimento, a continuidade de fenômeno de "preservação cerebral" encontrado no período fetal ${ }^{12,13}$. Este pressuposto baseia-se principalmente nos dados de diminuição relativa do IR cerebral em relação à circulação sistêmica nos fetos desnutridos ${ }^{13}$.

Este estudo não demonstrou diferença significativa tanto em relação ao IR e IP entre ACA e ACM. Diferentemente, a maioria dos estudos realizados em fetos tem demonstrado grande sensibilidade e especificidade em predizer o retardo no crescimento intrauterino pelo aumento do IR na artéria umbilical e o seu decréscimo nas artérias cerebrais, caracterizando assim o chamado efeito de centralização da circulação ${ }^{21}$. O IR é utilizado em grande parte dos estudos, como indicador de FSC ou, indiretamente, para quantificar a resistência vascular ${ }^{22}$.

A falta de poder do IR de Pourcelot em quantificar mudanças significativas da velocidade de FSC ou da resistência vascular periférica descrito em outros estudos $^{23,24}$, pode justificar os resultados deste trabaIho, onde as alterações de resistência vascular cerebral provavelmente tenham afetado de forma equivalente às velocidades sistólica e diastólica, produzindo valores maiores de VFPS e VFFD em RN AIG, porém sem diferença significativa no $I R$, em relação ao PIG. Por outro lado, um grande número de outros fatores e eventos pode afetar a circulação sanguínea intracraniana no período perinatal, além da condição nutricional, que é o objeto deste estudo. Infelizmente a maior parte dos estudos sobre a influência desta alteração foi e continua sendo realizada em fetos. No período pós-parto, além de dados escassos, a literatura tem mostrado valores bastante diversificados de fluxo cerebral, não só em relação à adequação peso/idade, mas também frente a outras variáveis ${ }^{12-16,25}$.

Neste contexto, tem sido descrito que a maturidade cronológica, especialmente a partir da $40^{\mathrm{a}}$ semana, assim como o maior peso de nascimento são fatores que influenciam no aumento das velocidades médias, além de propiciarem redução do IR e IP. As velocidades de fluxo são mais influenciadas pela idade gestacional do que pelo peso ao nascimento, graças à maturação dos vasos cerebrais, além de sofrerem grande influência da idade pós-natal (IPN) ${ }^{18,24}$. A IPN e a idade gestacional podem, portanto, influenciar significativamente a velocidade média, aumentando seus valores, e consequentemente, reduzindo os IR e IP conforme o grau de amadurecimento do RN. O peso de nascimento apresenta influência inversamente proporcional sobre o IR.

Nesta pesquisa não se observou diferença significativa de VMF na ACA com relação à idade gestacional provavelmente pela pequena variabilidade encontrada, por tratar-se de uma população de crianças a termo. Também não foi encontrada diferença em relação ao sexo, entretanto, apenas um estudo relatou que o sexo é fator de influência no FSC, com menor velocidade de FSC no sexo feminino, provavelmente por alterações hormonais e diferenças na viscosidade sanguínea e no débito cardíaco ${ }^{25}$.

Ao se observar a existência de uma associação entre valores médios de velocidade de FSC na ACA com a presença de policitemia, corroborou-se com resultados semelhantes observados por vários outros autores ${ }^{19,26,27}$, que também descreveram diminuição significativa dos valores de fluxo cerebral em RN com hiperviscosidade sanguínea. Os fatores etiológicos mais importantes para o seu desencadeamento provêm, em grande parte, da insuficiência placentária e de quadros de hipoxemia intra-uterina e o consequente aumento de eritropoiese fetal. Acomete com maior frequência $\mathrm{RN}$ com $\mathrm{RCIU}^{26}$, da forma como observado no presente estudo.

A razão das modificações do fluxo na policitemia neonatal é alvo de controvérsias, tendo sido correlacionadas a uma série de fatores e, não raramente, a uma associação deles, principalmente em relação ao aumento do conteúdo arterial de oxigênio, provocado pela elevação do hematócrito ou, como demonstrado experimentalmente através do uso de transfusões de sangue com metahemoglobina, produto do aumento da viscosidade do sangue ou do hematócrito ${ }^{26,27}$. Em outra análise, constatou-se que a PAM esteve associada aos valores de VMF em relação a ACA e ACM. No entanto, observa-se frequentemente na literatura que o fluxo cerebral em RNT não se associa à pressão arterial, ou seja, grande parte dos autores considera que o FSC dentro dos parâmetros de normalidade não é pressão-passiva ${ }^{28}$, embora não exista um consenso na literatura sobre 
os efeitos da PAS na hemodinâmica cerebral neonatal, inclusive com alguns autores considerando que a relação entre a pressão arterial e o fluxo forma um modelo denominado de caótico, pois se modifica continuamente em função de um grande número de co-variáveis ${ }^{29}$.

Pôde-se observar que os RN filhos de mães fumantes durante a gestação apresentaram valores de FSC diminuídos em ambos os territórios vasculares, com valores aumentados de IP, independente da condição de nutrição ao nascimento. No entanto, houve diferença significativa apenas em relação a VMF na ACM. Infelizmente são poucos os estudos a respeito dos efeitos imediatos do tabagismo nos valores de FSC e de vasos periféricos, sendo os resultados ainda conflitantes ${ }^{30}$.

De modo geral, o modelo de fluxo cerebral obtido no estudo mostrou-se diferente para cada uma das artérias. Estas diferenças mostram que o modelo de controle é bastante complexo, provavelmente sujeito à interdependência de outras variáveis não estudadas. Todos esses achados parecem demonstrar que são muitos os fatores que podem levar a alteração do padrão de fluxo em recém-nascidos, embora o RCIU pareça exercer um papel significativo, sendo possível também aventar a hipótese de respostas fisiológicas cerebrais diferentes em função do tipo e gravidade do retardo de crescimento intra-uterino.

\section{CONCLUSÕES}

Recém-nascidos PIG mostraram diminuição da velocidade de fluxo sanguíneo cerebral nos dois territórios avaliados, sendo significativamente diminuídos os valores de velocidade no pico sistólico e médio na ACA.

A velocidade média de fluxo foi influenciada de forma significativa pela adequação peso/idade gestacional, presença de policitemia neonatal e valores de pressão arterial média em ACA, sendo menor entre os recém-nascidos PIG e policitêmicos.

A presença de sofrimento fetal, valores de pressão arterial média e o tabagismo durante a gestação estiveram associados ao fluxo médio na ACM.

\section{REFERÊNCIAS}

1. Arend FB, Christa E, Heinz FRP. Intrauterine growth retardation, general movements, and neurodevelopmental outome: a review. Dev Med Child Neurol 2001;43:61-68.

2. Doctor BA, Oriordan MA, Kirchener HL, Shah D, Hack M. Perinatal correlates and neonatal outcomes of small for gestational age infants born at term gestation. Am J Obstet Gynecol 2001;185:652-659.

3. Hill RM, Vernaraud WM, Deter RL. The effect of intrauterine malnutrition on the term infant. Acta Paediatr Scand 1984;73:482-487.
4. Ounsted M, Moar VA, Scott A. Small for dates babies, gestational age and developmental ability at age 7 years. Early Hum Dev 1989;19:77-86.

5. Gutbrod T, Wolke D, Sohene B, Ohrt B, Riegel K. Effects of gestation and birth weight on the growth and development of very low birthweight small for gestational age infants: a matched group comparixson. Arch Dis Child Fetal Neonatal 2000;82:208-214.

6. Gherpelli JLD, Ferreira H, Costa HPF. Neurological follow-up of smallfor-gestational age newborn infants: a study of risk factors related to prognosis at one year of age. Arq Neuropsiquiatr 1993;51:50-58.

7. Lou HC, Skov H, Pedersen H. Low cerebral blood flow: a risk factor in the neonate. J Pediatr 1979;95:606-609.

8. Lubchenco LO, Hansman C, Boyd E. Intrauterine growh in length and head circunference as estimated from live births at gestational ages from 26 to 42 weeks. Pediatrics 1966;37:403-408.

9. Pezzati M, Dani C, Biadaioli R, et al. Early postnatal doppler assessment of cerebral blood flow velocity in healthy preterm and term infants. Dev Med Child Neurol 2002;44:745-752.

10. Capurro H, Konichezky S, Fonseca D, Caldeyro-Barcia R. A simplified method for diagnosis of gestational age in th newborn infant. J Pediatr 1978;93:120-122.

11 Lockwood CJ, Weiner S. Intrauterine fetal growth. Clin Perinatol 1986;13:3-35.

12. Scherjon SA, Oosting H, Kok JH, Zondervan HA - Effect of fetal brainsparing on the early neonatal cerebral circulation. Arch Dis Child 1994;71:11-15.

13. Madazli R, Uludag S, Ocak V. Doppler assessment of umbilical artery, thoracic aorta and middle cerebral artery in the management of pregnancies with growth restriction. Acta Obstet Gynecol Scand 2001;80:702-707.

14. Martinussen M, Brubakk AM, Vik T, Yao AC. Relationship between intrauterine growth retardation and early posnatal superior mesenteric artery blood flow velocity. Biol Neonate 1997;71:22-30.

15. Scherjon SA, KokJH, Oosting H, Wolf $\mathrm{H}$, Zondervan HA. Fetal and neonatal cerebral circulation: a pulsed study. J Perinat Med 1992; 20: 79-82.

16. Koons AH, Wurtzel D, Metcalf JM. Cerebral blood flow measurement in the newborn dog. Biol Neonate 1993;63:120-128.

17. Mari G, Russel LD. Middle cerebral artery flow velocity waveforms in normal and small for gestational age fetuses. Am J Obstet Gynecol 1992;166:1262-1270.

18. Ley D, Marsal AL. Doppler velocimetry in cerebral vessels of small for gestational age infants. Early Hum Dev 1992;31:171-180.

19. Netto AA, Brenelli-Vitali MA - Cerebral blood flow in policytemic newborn. Abstracts of the 5th World Congress of Perinatal Medicine. 2001 September 23-27; Barcelona, Spain. 302-304.

20. Perlman JM, Volpe JJ. Cerebral blood flow velocity in relation to intraventricular hemorrhage in the preterm newborn infant. J Pediatr 1982;100:956-959.

21. Sterne G, Shileds LE, Dubinsky TJ. Abnormal fetal cerebral and umbilical Doppler measurements in fetuses with intrauterine growth restriction predicts the severity of perinatal morbidity. J Clin Ultrasound 2001;29:146-151.

22. Okumura A, Toyota N, Hayakawa F, et al. Cerebral hemodynamics during early neonatal period in preterm infants with periventricular leukomalacia. Brain Dev 2002;24:693-697.

23. Assis MC, Machado HR. Medida da velocidade de fluxo nas artérias cerebrais utilizando ultra-som Doppler transfontanela antes e após o tratamento cirúrgico da hidrocefalia. Arq Neuropsiquiatr 1999;57:827-835.

24. Taylor GA, Short BL, Walker LK, Traysman RJ. Intracranial blood flow: quantification with duplex doppler and color doppler flow us. Radiology 1990;176:231-236.

25. Baenziger O, Jaggi JL, Mueller AC, et al. Cerebral blood flow in preterm infants affected by sex, mechanical ventilation and intrauterine growth. Pediatr Neurol 1994;11:319-324.

26. Black VD, Lubchenco LO - Neonatal polycythemia and hyperviscosity. Pediatr Clin N Am 1982;29:1137-1148.

27. Liem KD, Hopman JC, Oeseburg B, Haan AF, Kollee LA. The effect of blood transfusion and haemodilution on cerebral oxigenation and haemodynamics in newborn infants investigated by near infrared spectrophotometry. Eur J Pediatr Neurol 1997;156:305-310.

28. Tyszczuk L, Meek J, Elwell C, Wyatt JS - Cerebral blood flow is independent of mean arterial blood pressure in preterm infants undergoing intensive care. Pediatrics 1998;102:337-341.

29. Panerai RB, Simpson DM, Deverson ST, Mahony P, Hayes P, Evans $\mathrm{DH}$ - Multivariate dynamic analysis of cerebral blood flow regulation in humans. IEEE Trans Biomed Eng 2000;47:419-423.

30. Morioka C, Kondo H, Akashi K, et al. The continuos and simultaneous blood flow velocity measurement of four cerebral vessels and a peripheral vessel during cigarette smoking. Psychopharmacology 1997;131:220-229. 